\title{
Positive and Negative Donor Impurity in an InAs/AlAs Quantum Wire
}

\author{
N. Arunachalam ${ }^{1}$ and A. J. Peter ${ }^{2 *}$ \\ 'Department of Physics, Government Higher Secondary School, Saruguvalayapatti, Melur-625106, \\ India \\ *Department of Physics, Government Arts and Science College, Melur-625109, Madurai, India
}

Received 5 April 2010, accepted in final revised form 10 August 2010

\begin{abstract}
Binding energies of positive and negative charged donor impurities in an InAs/AlAs cylindrical quantum wire are investigated. Numerical calculations are performed using the variational procedure within the single band effective mass approximation. We assume that the impurity is located at the axis of the wire. The interband optical transition with and without the exciton is computed as a function of wire radius. The valence-band anisotropy is included in our theoretical model by using different hole masses in different spatial directions. Neutral shallow donors comprise a positively charged donor and a single bound electron. It is observed that (i) negative trions have a higher binding energy than positive trions, (ii) the binding energy of the heavy-hole exciton is much larger than that of the lighthole exciton due to different hole mass values (iii) the exciton binding energy and the interband emission energy are both increased when the radius of the cylindrical quantum wire is decreased and (iv) the effect of exciton influences the interband emission energy. Our results are in good agreement with the recent published results.
\end{abstract}

Keywords: Quantum wire; Impurity level; Binding energy; Excitons.

(c) 2010 JSR Publications. ISSN: 2070-0237 (Print); 2070-0245 (Online). All rights reserved.

DOI: 10.3329/jsr.v2i3.4715 J. Sci. Res. 2 (3), 433-441 (2010)

\section{Introduction}

Nano-structures of reduced dimensionality are currently a topic of interest in low dimensional semiconductors. Due to the recent techniques in fabricating low dimensional semiconductor systems, such as molecular beam epitaxy, chemical vapour deposition and electron lithography, the growth of low-dimensional quantum structures has attracted much attention recently. Production of self assembled monolayers of low dimensional semiconductors is particularly attractive due to its simplicity, versatility and low cost [1]. Self-assembled InAs quantum wires embedded in AlAs barriers are of interest because of their large confinement potential compared to InAs/AlAs quantum wells [2]. It has

\footnotetext{
*Corresponding author: a.john.peter@gmail.com
} 
attracted much attention due to their electronic, optical properties and potential for applications.

The impurity plays an elementary role in some physical properties like optical, electrical and transport phenomena at low temperature. The main objective of addition of impurities in semiconductor heterostructures lies in altering the performance of quantum devices. In order to investigate quantum size effects on exciton states and optical properties of InAs/AlAs nanostructures, we have investigated the relationship between donor bound exciton states and the interband emission in an InAs/AlAs quantum wire by using a variational approach. InAs quantum wires in AlAs differ considerably from that in GaAs due to the larger Al-In bond strength, reducing In migration on the AlAs surface, leading to a larger density and smaller size of InAs/AlAs quantum wires [3].

Optical transitions in coupled InAs/InP self-assembled quantum wires have been studied within the single-band effective mass approximation including strain effects by Sidor et al., recently [4]. The photoluminescence (PL) spectra of InAs/GaAs quantum dots under the hydrostatic pressure were examined experimentally [5, 6]. Duque et al. [7] have studied the hydrostatic pressure effects theoretically on optical transitions in InAs/GaAs cylindrical quantum dots. These studies reveal the exact shape and dimensions of InAs/GaAs quantum dots clearly. The donor binding energy of a hydrogenic impurity in InAs/GaAs single quantum dot as functions of impurity position, size and hydrostatic pressure has been computed [8]. Bracker et al. [9] have compared binding energies for positive and negative trions in a series of narrow GaAs quantum wells and in "natural" quantum dots defined by quantum well thickness fluctuations recently and they have observed that negative trions have a higher binding energy than positive trions. The study of positive and negative trions with exact diagonalization in the influence of magnetic field has been also carried out recently [10].

In this paper, we calculate the binding energies of positive and negative charged donor impurities as a function of radius of cylindrical quantum wire of InAs/AlAs. Numerical calculations have been performed using the single band effective mass approximation with the variational procedure assuming the impurity is located at the axis of wire. The interband optical transition of InAs/AlAs quantum wire is also computed as a function of wire radius. The valence-band anisotropy is included in our theoretical model by using different hole masses in different directions. This work is organized as follows. In Section 2, we present the theoretical model to describe $D^{0}$ (neutral donor), $D^{+}$(positive donor) and $D^{-}$(negative donor) states confined in an InAs quantum wire. Results are discussed in Section 3 and at the end we discuss our findings.

\section{Theory and Model}

\subsection{Binding energy of neutral donor $D^{0}$}

We consider a cylindrical quantum wire of radius $R$ and length $L$, made of InAs surrounded by AlAs and the electron is confined by the potential $V_{e}\left(\rho_{j}, z_{j}\right)$ equals zero inside the quantum wire (InAs) and $V_{e}^{0}$ in the barrier material. $D^{0}$ is described by the 
system of an electron attached to a positively charged donor impurity ion located on the wire axis. In the frame work of single band effective mass approximation, the Hamiltonian of a hydrogenic donor can be written as

$$
H_{D^{0}}=-\frac{\hbar^{2}}{2 m_{e}^{*}} \nabla_{e}^{2}+V_{e}\left(\rho_{e}, z_{z}\right)-\frac{e^{2}}{\varepsilon \sqrt{\rho^{2}+z^{2}}}
$$

where $m_{e}^{*}$ is the effective mass of electron and $\varepsilon$ is the static dielectric constant of the host material.

The electron (hole) confinement potential $V\left(\rho_{j}, z_{j}\right)$ due to the band offset in the InAs/AlAs quantum wire structure is given by

$$
V\left(\rho_{j}, z_{j}\right)= \begin{cases}0 & \rho_{j} \leq R \\ V_{j}^{0} & \text { otherwise }\end{cases}
$$

where the index $j$ stands for electron and hole and $V^{0}$ is the barrier height. We have taken $V_{e}^{0}=2175 \mathrm{meV}$ and $V_{h}^{0}=611 \mathrm{meV}[11]$.

In order to find the eigenvalue for Hamiltonian (Eq.(1)), we apply the variational method with the trial wave function

$$
\psi_{D^{0}}\left(\rho_{j}, \varphi_{j}, z_{j}\right)=f\left(\rho_{j}\right) h\left(z_{j}\right) \chi\left(\rho_{j}, z_{j}\right) e^{i m \varphi_{j}}
$$

where $m$ is the electron (hole) $z$-component angular momentum quantum number. The radial wave function $f\left(\rho_{j}\right)$ and the corresponding confinement energy equation of the electron (hole) can be obtained by using the $m$-order Bessel function $J_{m}$ and the modified Bessel function $K_{m}$. The $z$-axis wave function $h\left(z_{j}\right)$ can be found using linear combinations of analytical functions $\sin (\xi)$ and $\cos (\xi)$ (inside the dot), or $\exp (\xi)$ (outside the dot), with the single parameter trial wave function chosen as

$$
\chi\left(\rho_{e}, \rho_{h}, z_{e}, z_{h}\right)=\exp \left(-\alpha_{1} \sqrt{\rho^{2}+z^{2}}\right)
$$

where $\alpha_{I}$ is the variational parameter.

The ground state energy of a single electron confined by the donor impurity ion located along the wire axis is obtained by the expectation value of the energy of the Hamiltonian as

$$
\left\langle E_{D^{0}}\right\rangle=\min \frac{\left\langle\psi_{D^{0}}\left|H_{D^{0}}\right| \psi_{D^{0}}\right\rangle}{\left\langle\psi_{D^{0}} \mid \psi_{D^{0}}\right\rangle}
$$

The donor binding energy is obtained by 


$$
E_{b}\left(D^{0}\right)=E-\left\langle E_{D^{0}}\right\rangle
$$

where $E$ is the ground state energy of the electron.

Table 1. Material parameters used in the calculations.

(values from refs. [14] and [15]).

\begin{tabular}{|c|c|c|}
\hline Parameter & InAs & AlAs \\
\hline$E_{g}(\mathrm{eV})$ & 0.417 & 2.15 \\
$m_{e}$ & 0.023 & 0.077 \\
$\gamma_{1}$ & 20 & 3.76 \\
$\gamma_{2}$ & 8.5 & 0.82 \\
$\varepsilon$ & 15.15 & 10.06 \\
\hline
\end{tabular}

\subsection{Binding energy of positive donor $\mathrm{D}^{+}$}

The Hamiltonian for a positive donor can be thought of as a neutral donor and an excess hole. Therefore, we can write

$$
H_{D^{+}}=H_{e}+H_{h}+V_{C}
$$

where $H_{e}$ refers the Hamiltonian of the single electron part, $H_{h}$ is the Hamiltonian of the single hole part with the electron and hole confinement given by the Eq.(2). The last term, $V_{C}$, is the Coulomb interaction between the electron-hole, the positive ion-hole and the positive ion-electron interactions respectively, given by

$$
V_{C}=+\frac{e^{2}}{\varepsilon \sqrt{\rho_{e}^{2}+z_{e}^{2}}}+\frac{e^{2}}{\varepsilon \sqrt{\rho_{h}^{2}+z_{h}^{2}}}-\frac{e^{2}}{\varepsilon \sqrt{\left(\rho_{e}-\rho_{h}\right)^{2}+\left(z_{e}-z_{h}\right)^{2}}}
$$

The anisotropy and the corrections due to the conduction band non-parabolicity are important since the parallel mass determines the electron energy in the $x y$-plane and perpendicular mass determines the quantization energy of the electron in the $z$-direction. In the valence band of semiconductors, there are heavy hole and light hole bands which are degenerate at the top of the valence band. The valence-band anisotropy is described by assuming different hole masses in different directions. The heavy-hole and light-hole masses in terms of Luttinger parameters, $\gamma_{1}$ and $\gamma_{2}$, (Table 1 ) are given by [12]

$$
\frac{m_{0}}{m_{h h}^{*}}=\gamma_{1}-2 \gamma_{2}
$$




$$
\frac{m_{0}}{m_{l h}^{*}}=\gamma_{1}+2 \gamma_{2}
$$

here $m_{0}=$ is the free hole mass. The corresponding wave function for the positive donor impurity $D^{+}$is given by

$$
\psi_{D^{+}}\left(\rho_{e}, \rho_{h}, z_{e}, z_{h}\right)=f\left(\rho_{e}\right) f\left(\rho_{h}\right) g\left(z_{e}\right) g\left(z_{h}\right) \chi\left(\rho_{e}, \rho_{h}, z_{e}, z_{h}\right)
$$

where $\chi$ is the trial wave function describing the internal motion between the electron and the hole in the system, given by

$$
\chi\left(\rho_{e}, \rho_{h}, z_{e}, z_{h}\right)=\exp \left(-\alpha_{2} \sqrt{\rho^{2}+z^{2}}\right)
$$

where $\alpha_{2}$ is the variational parameter. We calculate the ground state energy by finding out the expectation value of the energy of the Hamiltonian (Eq.(7)) as

$$
\left\langle E_{D^{+}}\right\rangle=\min \frac{\left\langle\psi_{D^{+}}\left|H_{D^{+}}\right| \psi_{D^{+}}\right\rangle}{\left\langle\psi_{D^{+}} \mid \psi_{D^{+}}\right\rangle}
$$

The binding energy of the positive charged donor, $E_{b}\left(D^{+}\right)$, is given by

$$
E_{b}\left(D^{+}\right)=E_{h}+E_{D^{0}}-\left\langle E_{D^{+}}\right\rangle
$$

where $E_{h}$ is the ground state energy of the free hole.

\subsection{Binding energy of negative donor $\mathrm{D}^{-}$}

The Hamiltonian of negative donor can be thought of as a neutral donor and an excess electron

$$
H_{D^{-}}=H_{e 1}+H_{e 2}+V_{C}
$$

Here the Coulomb interaction is between the electron-electron, the positive ion-hole and the positive ion-electron respectively. $V_{c}$ can be expressed as

$$
V_{C}=-\frac{e^{2}}{\varepsilon \sqrt{\rho_{e 1}^{2}+z_{e 1}^{2}}}-\frac{e^{2}}{\varepsilon \sqrt{\rho_{e 2}^{2}+z_{e 2}^{2}}}+\frac{e^{2}}{\varepsilon \sqrt{\left(\rho_{e 1}-\rho_{e 2}\right)^{2}+\left(z_{e 1}-z_{e 2}\right)^{2}}}
$$

Thus the binding energy of the negative charged donor, $E_{b}\left(D^{-}\right)$given by

$$
E_{b}\left(D^{-}\right)=E_{e}+E_{D^{0}}-\left\langle E_{D^{-}}\right\rangle
$$


where $E_{e}$ is the ground state energy of the free electron. $E_{D^{-}}$is calculated by minimizing $H_{D^{+}}$using the similar trial wave function as given by Eq. (12) but with different the variational parameter $\alpha_{3}$.

\subsection{Interband emission energy, $E_{p h}$}

The Hamiltonian of an exciton consisting of a single electron part $\left(H_{e}\right)$, the single hole part $\left(H_{h}\right)$ and Coulomb attraction term between electron-hole pair is given by,

$$
H_{e x}=H_{e}+H_{h}-\frac{e^{2}}{\varepsilon|r|}
$$

where $|\bar{r}|=\left|\bar{r}_{e}-\bar{r}_{h}\right|$ denotes the relative distance between the electron and the hole and $\varepsilon$ is the dielectric constant of material inside the quantum wire.

The ground state energy of the exciton in the InAs/AlAs quantum wire is calculated by using the following equation

$$
E_{e x}=\min _{\alpha, \beta} \frac{\left\langle\psi_{e x}\left|H_{e x}\right| \psi_{e x}\right\rangle}{\left\langle\psi_{e x} \mid \psi_{e x}\right\rangle}
$$

The donor exciton binding energy $E_{b}$ and the interband emission energy $E_{p h}$ associated with the exciton is calculated using the following equations

$$
\begin{aligned}
& E_{b}=E_{e}+E_{h}-E_{e x} \\
& E_{p h}=E_{e}+E_{h}+E_{g}-E_{e x}
\end{aligned}
$$

where $E_{e}$ and $E_{h}$ are the confinement energies of the electron and hole, respectively. $E_{g}$ is the band gap energy of InAs.

\section{Results and Discussion}

We show the variation of binding energy of negative and positive donor impurity as a function of wire radius in Fig. 1. In both the cases, the binding energy increases with a decrease of wire radius, reaching a maximum value and then decreases when the wire radius still decreases. As the wire radius approaches zero the confinement becomes negligibly small, and in the finite barrier problem the tunnelling becomes huge. The increase of negative charged impurity binding energy with the wire radius is due to the Coulomb attraction between the electron and hole and interaction between each one of them and the impurity ion. The increase of negative donor binding energy with the decrease of wire radius is in good agreement with the other investigators [13]. The values 
of positive donor binding energy $E_{b}\left(D^{+}\right)$drop faster than the negative donor binding energy. This is due to the repulsion between two electrons which is larger than the attraction of each electron with the impurity ion. Moreover, we have observed that negative trions are found to have a higher binding energy than positive trions, which is in good agreement with other studies [9,10]. Also it is observed from the figures that $\mathrm{D}^{-}$ compound is less stable than the $\mathrm{D}^{+}$compound.

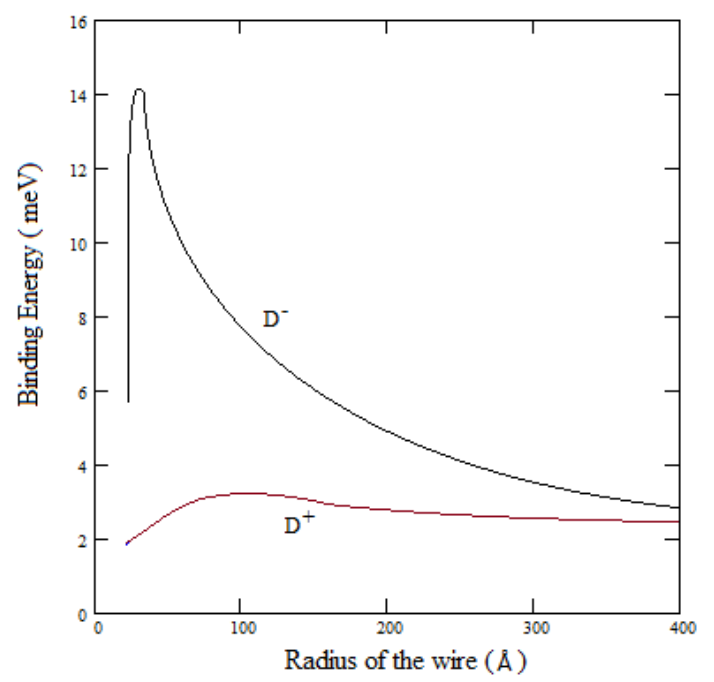

Fig. 1. Variation of binding energy of negative and positive donor impurities as a function of wire radius.

Fig. 2 shows the variation of the binding energy of positive donor impurity for both heavy and light holes as a function of wire radius. In both the cases, we notice that the positive donor binding energy increases as the wire radius decreases and then reaches the maximum value and starts decreasing when the radius of wire still decreases, it is due to tunnelling effect. The binding energy of the heavy-hole exciton is much larger than that of the light-hole exciton due to its larger effective mass.

We display the variation of interband emission energy as a function of wire radius in Fig. 3. This representation clearly brings out the quantum size effect. It is noted that the interband emission energy decreases monotonically as the wire radius increases. This is due to the confinement of electron-hole with respect to $z$-axis when the wire radius increases. The Coulomb interaction is thus reduced implementing the exciton binding energy which is an indication of the electron-hole Coulomb interaction. Moreover it is clearly shown that the effect of exciton has influence on the interband emission energy. 


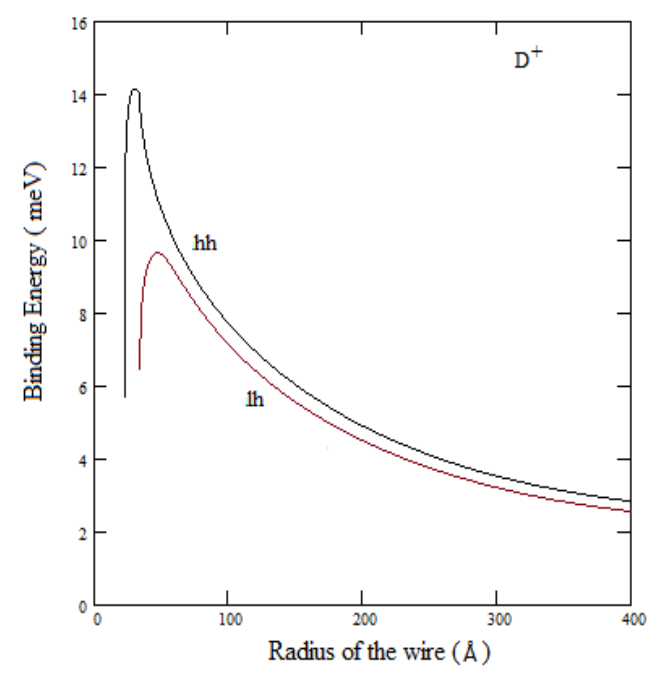

Fig. 2. Variation of binding energy of positive donor impurity for both heavy and light hole as a function of wire radius.

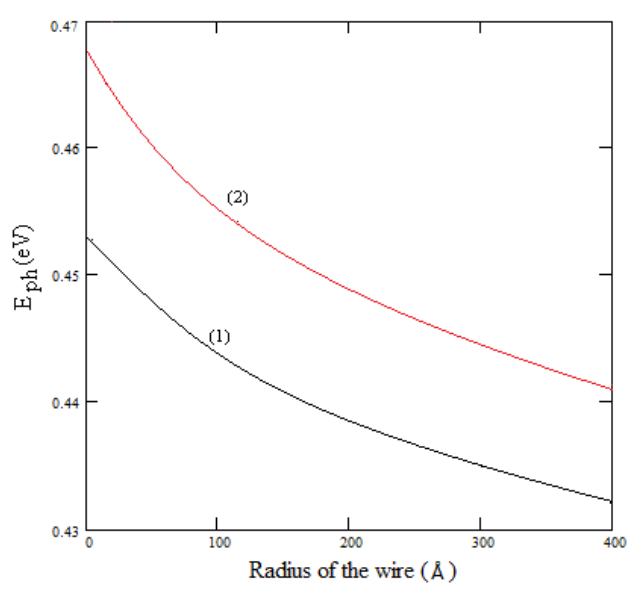

Fig. 3. Variation of interband emission energy, $E_{p h}$, as a function of wire radius.

The curve (2) shows the effect without the exciton.

In conclusion, we have studied the positive and negative binding energies of donor impurities in the InAs/AlAs quantum wire. The calculations have been carried out within the framework of effective mass approximation using the variational approach. We have obtained the binding energies for $D^{+}$and $D^{-}$as a function of wire radius taking into account of the valence-band anisotropy through energy dependent effective mass in different spatial directions. Numerical results show that the positive donor binding energy 
increases when the wire radius decreases and the exciton binding energy and the interband emission energy both increase when the radius of cylindrical quantum wire is decreased. The heavy hole compound is more bound than the light hole compound. The effect of exciton has influence on the interband emission energy. Negative trions are found to have a higher binding energy than positive trions. We hope that our investigations would play an important role in designing opto-electronic devices based on InAs/AlAs quantum wire structures.

\section{Acknowledgement}

One of the authors (AJP) thanks the University Grants Commission, India, for the grant, MRP (F.No.38-78/2009( (SR)) to support this work.

\section{References}

1. D. S. L. Mui, D. Leonard, L. A. Coldren, and P. M. Petroff, Appl. Phys. Lett. 66, 13 (1995). doi:10.1063/1.113871

2. R. Notzel, J. Temmyo, and T. Tamamura, Appl. Phys. Lett. 64, 3557 (1994). doi:10.1063/1.111196

3. Pierz, Z. Ma, U. F. Keyser, and R. J. Haug, J. Cryst. Growth 249, 477 (2003). doi:10.1016/S0022-0248(02)02333-3

4. Y. Sidor, B. Partoens, F. M. Peeters, T. Ben, A. Ponce, D. L. Sales, S. I. Molina, D. Fuster, L. Gonz'alez, and Y. Gonz'alez, Phys. Rev. B 75, 125120 (2007).

doi:10.1103/PhysRevB.75.125120

5. I. E. Itskevich, S. G. Lyapin, I. A. Troyan, P. C. Klipstein, L. Eaves, P. C. Main, M. Henini, Phys. Rev. B 58, R4250 (1998). doi:10.1103/PhysRevB.58.R4250

6. B. S. Ma, X. D. Wang, F. H. Su, Z. L. Fang, K. Ding, Z. C. Niu, G. H. Li, J. Appl. Phys. 95, 933 (2004). doi:10.1063/1.1635988

7. C. A. Duque, N. Montenegro, Z. Barticevic, M. Pacheco, L. E. Oliveira, J. Phys.: Cond. Matter 18, 1877 (2006). doi:10.1088/0953-8984/18/6/005

8. C. X. Xia, Y. M. Liu, S. Y. Wei, Appl. Surf. Sci. 254, 3479 (2008).

9. A. S. Bracker, E. A. Stinaff, D. Gammon, M. E. Ware, J. G. Tischler, and D. Park, Phys. Rev. B 72, 035332 (2005). doi:10.1103/PhysRevB.72.035332

10. A. Trojnar and A. WÓjs, Proc. XXXVII International School of Semiconducting Compounds, Jaszowiec 114, 1411 ( 2008).

11. P. Offermans, P. M. Koenraad, and J. H. Wolter, K. Pierz M. Roy and P. A. Maksym, Phys. Rev. B 72, 165332 (2005). doi:10.1103/PhysRevB.72.165332

12. J. Simon, N. T. Pelekanos, C. Adelmann, E. M. Guerrero, B. Daudin, L. S. Dang, H. Mariette, Phys. Rev. B 68, 035312 (2003). doi:10.1103/PhysRevB.68.035312

13. S. Baskoutas, W. Schommers, A. F. Terzis, V. Kapaklis, M. Rieth and C. Politis, Phys. Lett. A 308, 219 (2003). doi:10.1016/S0375-9601(03)00025-2

14. I. Vurgaftman and J. R. Meyer and L. R. Ram-Mohan, J. Appl. Phys. 89, 5815 (2001).

15. S. Adachi, J. Appl. Phys. 58, 1 (1985). doi:10.1063/1.336070 